\title{
AIDS-Related Immunoblastic Lymphoma
}

National Cancer Institute

\section{Source}

National Cancer Institute. AIDS-Related Immunoblastic Lymphoma. NCI Thesaurus.

Code C8285.

A morphologic variant of diffuse large B-cell lymphoma occurring in HIV-positive patients. 